Document downloaded from:

http://hdl.handle.net/10251/82363

This paper must be cited as:

Beltran, A.; Felipe Román, MJ.; Melchor, C. (2016). Normal subgroups whose conjugacy class graph has diameter three. Bulletin of the Australian Mathematical Society. 94(2):266272. doi:10.1017/S0004972715001860.

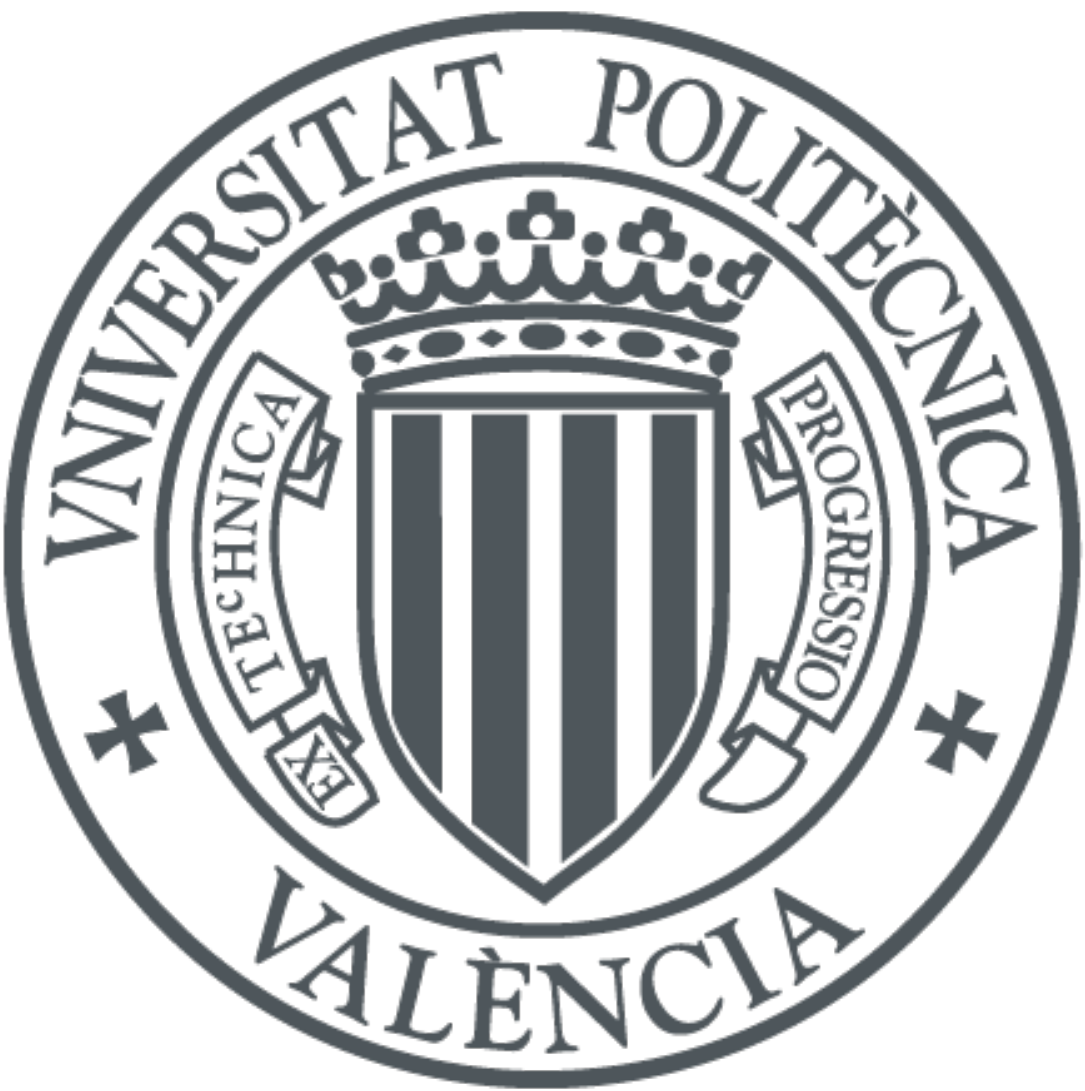

The final publication is available at

http://doi.org/10.1017/S0004972715001860

Copyright Cambridge University Press (CUP)

Additional Information 


\title{
Normal SUbGROUPS WHOSE CONJUGACY CLASS GRAPH HAS DIAMETER THREE.
}

\author{
Antonio Beltrán \\ Departamento de Matemáticas, \\ Universidad Jaume I, 12071 Castellón, Spain \\ e-mail: abeltran@mat.uji.es \\ María José Felipe \\ Instituto Universitario de Matemática Pura y Aplicada, \\ Universidad Politécnica de Valencia, 46022 Valencia, Spain \\ e-mail: mfelipe@mat.upv.es \\ Carmen Melchor \\ Departamento de Educación, \\ Universidad Jaume I, 12071 Castellón, Spain \\ e-mail: cmelchor@uji.es
}

\begin{abstract}
Let $G$ be a finite group and $N$ a normal subgroup of $G$. We determine the structure of $N$ when the graph associated to the $G$-conjugacy classes contained in $N$ has diameter three.
\end{abstract}

Keywords. Finite groups, conjugacy classes, normal subgroups, graphs. Mathematics Subject Classification (2010): 20E45, $20 \mathrm{D} 15$.

\section{Introduction}

Let $G$ be a finite group and let $N$ be a normal subgroup of $G$ and let $x \in N$. We denote by $x^{G}=\left\{x^{g} \mid g \in G\right\}$ the $G$-conjugacy class of $x$. Let $\Gamma_{G}(N)$ be the graph associated to these $G$-conjugacy classes, which was defined in [2] as follows: its vertices are the $G$-conjugacy classes of $N$ of cardinality bigger than 1, that is, $G$-classes of elements in $N \backslash(\mathbf{Z}(G) \cap N)$, and two of them are joined by an edge if their sizes are not coprime. It was proved in [2] that $d\left(\Gamma_{G}(N)\right) \leq 3$ where $d\left(\Gamma_{G}(N)\right)$ denotes the diameter of the graph. In this paper we analyze 
the structure properties of $N$ when $d\left(\Gamma_{G}(N)\right)=3$.

The above graph extends the ordinary graph, $\Gamma(G)$, which was formerly defined in [3], whose vertices are the non-central conjugacy classes of $G$ and two vertices are joined by an edge if their sizes are not coprime. The graph $\Gamma_{G}(N)$ can be viewed as the subgraph of $\Gamma(G)$ induced by those vertices of $\Gamma(G)$ which are vertices in $\Gamma_{G}(N)$. This fact does not allow to obtain directly properties of the graph of $G$-classes.

Concerning ordinary classes, L.S. Kazarin characterizes in [7] the structure of a group $G$ having two "isolated classes". Remember that a group $G$ has isolated conjugacy classes if there exist elements $x, y \in G$ with coprime conjugacy class sizes such that every element of $G$ has conjugacy class size coprime to either $\left|x^{G}\right|$ or $\left|y^{G}\right|$. Particularly Kazarin determined the structure of the groups $G$ with $d(\Gamma(G))=3$. It should be noted that similar results have also been tested for other graphs. In [5], Dolfi defines the graph $\Gamma^{\prime}(G)$ whose vertices are the elements of the set of all primes which occur as divisors of the lengths of the conjugacy classes of $G$, and two vertices $p, q$ are joined by an edge if there exists a conjugacy class in $G$ whose length is a multiple of $p q$. In [6] Dolfi and Casolo describe all finite groups $G$ for which $\Gamma^{\prime}(G)$ is connected and has diameter three.

We have to remark that the primes dividing the $G$-conjugacy class sizes not necessarily divide $|N|$, it can occur the case when $N$ is abelian and it is noncentral in $G$ and consequently we have not control on these primes. For this reason, we observe that new cases appear when we work with $G$-classes which are not contemplated in the ordinary case. The main result of this paper is the following theorem. From now on, if $H$ is a subgroup of a finite group $G$ we denote by $\pi(H)$ the set of primes dividing $|H|$.

Theorem A. Let $G$ be a finite group and $N \unlhd G$. Suppose that $x^{G}$ and $y^{G}$ are two non-central $G$-conjugacy classes of $N$ such that any $G$-conjugacy of $G$ has size coprime with $\left|x^{G}\right|$ or $\left|y^{G}\right|$. Let $\pi_{x}=\pi\left(\left|x^{G}\right|\right), \pi_{y}=\pi\left(\left|y^{G}\right|\right)$ and $\pi=\pi_{x} \cup \pi_{y}$. Then, $N=\mathbf{O}_{\pi^{\prime}}(N) \times \mathbf{O}_{\pi}(N)$ with $x, y \in \mathbf{O}_{\pi}(N)$ which is a quasiFrobenius group with abelian kernel and complement or $\mathbf{O}_{\pi}(N)=P \times A$ with $A \leq \mathbf{Z}(N)$ and $P$ a p-group for a prime $p$.

Notice that in the conditions of Theorem A if $d\left(\Gamma_{G}(N)\right) \leq 2$ it follows that the graph is disconnected and the structure of $N$ is determined in Theorem $\mathrm{E}$ of [2]. Consequently, $d\left(\Gamma_{G}(N)\right)=3$ and we obtain the following result.

Corollary. Let $G$ be a finite group and $N \unlhd G$. Suppose that $\Gamma_{G}(N)$ is connected and $d\left(\Gamma_{G}(N)\right)=3$. Let us consider $x, y \in N$ such that $d\left(x^{G}, y^{G}\right)=3$. Set $\pi=\pi\left(\left|x^{G}\right|\right) \cup \pi\left(\left|y^{G}\right|\right)$. We have that $x, y \in \mathbf{O}_{\pi}(N), N=\mathbf{O}_{\pi^{\prime}}(N) \times \mathbf{O}_{\pi}(N)$ with $\mathbf{O}_{\pi}(N)$ a quasi-Frobenius group with abelian kernel and complement or $\mathbf{O}_{\pi}(N)=P \times A$ with $A \leq \mathbf{Z}(N)$ and $P$ a p-group for a prime $p$. 
Proof. It follows immediately by Theorem A.

Proofs of these results are based on the techniques appeared in [7] although we do not use them in ours. When $N=G$ we obtain the result of Kazarin.

\section{Proof of Theorem A}

First, we show three elementary results necessary to prove the main theorem.

Lemma 1. Let $G$ a $\pi$-separable group. Then the conjugacy class length of every $\pi$-element of $G$ is a $\pi$-number if and only if $G=H \times K$, where $H$ and $K$ are a Hall $\pi$-subgroup and a $\pi$-complement of $G$, respectively.

Proof. This is Lemma 8 of [1].

In the particular case in which $\pi=p^{\prime}$, the complement of some prime $p$, the above Lemma is true without assuming $p$-separability (which is equivalent to $p$-solvability).

Lemma 2. If, for some prime $p$, every $p^{\prime}$-element of a group $G$ has index prime to $p$, then the Sylow p-subgroup of $G$ is a direct factor of $G$.

Proof. This is Lemma 1 of [4].

Lemma 3. Let $G$ be a finite group and $N \unlhd G$. Let $B=b^{G}$ and $C=c^{G}$ be two non-central $G$-conjugacy classes of $N$. If $(|B|,|C|)=1$. Then

a. $\mathbf{C}_{G}(b) \mathbf{C}_{G}(c)=G$.

b. $B C=C B$ is a non-central $G$-class of $N$ and $|B C|$ divides $|B||C|$.

c. Suppose that $d(B, C) \geq 3$ and $|B|<|C|$. Then $|B C|=|C|$ and $C B B^{-1}=$ $C$. Furthermore, $C\left\langle B B^{-1}\right\rangle=C,\left\langle B B^{-1}\right\rangle \subseteq\left\langle C C^{-1}\right\rangle$ and $\left|\left\langle B B^{-1}\right\rangle\right| d i$ vides $|C|$.

Proof. This is Lemma 2.1 of [2].

Proof of Theorem A. We proceed by induction on $|N|$. Notice that the hypotheses are inherited by every normal subgroup in $G$ which is contained in $N$ and contains $x$ and $y$. By using the primary decomposition we can assume that $x$ and $y$ have order a power of two primes, say $p$ and $q$, respectively.

Step 1. $q=p$ if and only if $x y=y x$.

Suppose that $x y=y x$ and that $p \neq q$. Observe that $\mathbf{C}_{G}(x y)=\mathbf{C}_{G}(x) \cap$ $\mathbf{C}_{G}(y)$ and consequently, $\left|x^{G}\right|$ divides $\left|(x y)^{G}\right|$ and $\left|y^{G}\right|$ divides $\left|(x y)^{G}\right|$. Thus, we 
obtain a $G$-conjugacy class connected with $x^{G}$ and $y^{G}$, which is a contradiction by hypotheses. Conversely, suppose that $p=q$. We know that $p$ cannot divide either $\left|x^{G}\right|$ or $\left|y^{G}\right|$. Furthermore, the hypotheses imply that $\left(\left|x^{G}\right|,\left|y^{G}\right|\right)=1$, so we have $G=\mathbf{C}_{G}(x) \mathbf{C}_{G}(y)$ and $\left|x^{G}\right|=\left|G: \mathbf{C}_{G}(x)\right|=\left|\mathbf{C}_{G}(y): \mathbf{C}_{G}(x) \cap \mathbf{C}_{G}(y)\right|$. Now, since $y$ is a $p$-element in $\mathbf{Z}\left(\mathbf{C}_{G}(y)\right)$, we deduce that $y \in \mathbf{C}_{G}(x) \cap \mathbf{C}_{G}(y)$ and hence $x y=y x$.

Step 2. $p, q \in \pi$.

We define $K=\mathbf{C}_{G}(x) \cap \mathbf{C}_{G}(y)$. First, we assume that $p \neq q$ and $x y \neq y x$. We have $|G: K|=\left|G: \mathbf{C}_{G}(x)\right|\left|\mathbf{C}_{G}(x): \mathbf{C}_{G}(x) \cap \mathbf{C}_{G}(y)\right|=\left|x^{G}\right|\left|y^{G}\right|$, which is a $\pi$-number. Since $x \in \mathbf{Z}\left(\mathbf{C}_{G}(x)\right)$ and $x$ is a $p$-element but $x \notin K$, we know that $p$ divides $\left|\mathbf{C}_{G}(x): K\right|=\left|y^{G}\right|$. This means that $p \in \pi_{y}$. Similarly we obtain that $q$ divides $\left|x^{G}\right|$, that is, $q \in \pi_{x}$. Consequently, $p, q \in \pi$.

Suppose now that $p=q$ and $x y=y x$. Let us see that $p \in \pi$. We denote $X=x^{G}$ and $Y=y^{G}$ and we assume for instance that $|X|>|Y|$. By hypothesis, the distance between $X$ and $Y$ in $\Gamma_{G}(N)$ is 3. We can apply Lemma 3(c) and we obtain $X\left\langle Y Y^{-1}\right\rangle=X,\left\langle Y Y^{-1}\right\rangle \subseteq\left\langle X X^{-1}\right\rangle$ and $\left|\left\langle Y Y^{-1}\right\rangle\right|$ divides $|X|$. On the other hand, since $G=\mathbf{C}_{G}(x) \mathbf{C}_{G}(y)$ we have $X \subseteq \mathbf{C}_{G}(y)$. As a result, $\left\langle Y Y^{-1}\right\rangle \subseteq\left\langle X X^{-1}\right\rangle \subseteq \mathbf{C}_{G}(y)$. In particular, if we take $z=y^{g} \neq y$, for some $g \in G$, we have $w=z y^{-1} \in\left\langle Y Y^{-1}\right\rangle \subseteq \mathbf{C}_{G}(y)$, so $[z, y]=1$. We obtain that $w$ is a non-trivial $p$-element and, since $p$ divides $\left|\left\langle Y Y^{-1}\right\rangle\right|$, which divides $|X|$, we conclude that $p \in \pi_{x}$. If $|Y|>|X|$ we can argue similarly to get $p \in \pi_{y}$.

Step 3. We can assume that $N / \mathbf{Z}(N)$ is neither a p-group nor a q-group (particularly, we can assume that $N$ is not abelian).

As we have said at the beginning, $x$ is a $p$-element and $y$ is a $q$-element. Suppose that $N / \mathbf{Z}(N)$ is a $p$-group (the reasoning is analogous if we suppose that it is a $q$-group). Hence we can write $N=P \times A$ where $A \leq \mathbf{Z}(N)$ and $A$ is a $p^{\prime}$-group. If $p \neq q$, it follows that $x \in P$ and $y \in A$, which leads to a contradiction with Step 1. Thus, $p=q$ and $x, y \in P$, so the theorem is proved.

Step 4. We can suppose that $N$ is not a $\pi$-group.

Let us see that if $N$ is a $\pi$-group, then $N$ is a quasi-Frobenius group with abelian kernel and complement or $N=P \times A$ with $A \leq \mathbf{Z}(N)$ and $A$ a $p^{\prime}$ group. Assume that $N$ is a $\pi$-group. As $N$ is non-abelian by Step 3 , there exists a conjugacy class $z^{N}$ such that $\left|z^{N}\right| \neq 1$, Since $\left|z^{N}\right|$ divides $\left|z^{G}\right|$, then either $\left(\left|z^{N}\right|,\left|x^{G}\right|\right)=1$ or $\left(\left|z^{N}\right|,\left|y^{G}\right|\right)=1$. Thus, $\left|z^{N}\right|$ either is a $\pi_{x}$-number or a $\pi_{y}$-number. If $\Gamma(N)$ is disconnected, we know by Theorem 2 of [3] that $N$ is quasi-Frobenius group with abelian kernel and complement. Moreover, $\Gamma(N)$ cannot be empty since by Step 3, we can assume that $N$ is not abelian. Consequently, we can assume that $\Gamma(N)$ is connected and this forces to either $\left|x^{N}\right|=1$ or $\left|y^{N}\right|=1$. Suppose for instance that $\left|x^{N}\right|=1$, that is, $x \in \mathbf{Z}(N)$. 
By Step 3 we can take $w$ an $s$-element of $N \backslash \mathbf{Z}(N)$ with $s \neq p$. Observe that $\left|w^{N}\right|$ must be a $\pi_{y}$-number, so $w^{G}$ is connected to $y^{G}$ in $\Gamma_{G}(N)$. Since $x$ and $w$ have coprime orders and $x \in \mathbf{Z}(N)$ we have that $\left|w^{G}\right|$ and $\left|x^{G}\right|$ both divide $\left|(w x)^{G}\right|$. As a consequence, we have a contradiction because $\left|(w x)^{G}\right|$ has primes in $\pi_{x}$ and $\pi_{y}$. Then we can suppose that $N$ is not a $\pi$-group.

\section{Step 5. Conclusion in case $p \neq q$.}

Let $z$ be a $\pi^{\prime}$-element of $K \cap N$ and let us prove that $\left|z^{G}\right|$ is a $\pi^{\prime}$-number. Suppose that $s \in \pi$ is a prime divisor of $\left|z^{G}\right|$. We can assume for instance that $s \in \pi_{y}$, otherwise we proceed analogously. Since $\left|z^{G}\right|$ divides $\left|(z x)^{G}\right|$ we obtain that $s$ divides $\left|(z x)^{G}\right|$. On the other hand, we know by the proof of Step 2 that $q \in \pi_{x}$. Therefore, $\left|(z x)^{G}\right|$ is divisible by primes in $\pi_{x}$ and $\pi_{y}$, a contradiction. Consequently, $s \notin \pi$ and $\left|z^{G}\right|$ is a $\pi^{\prime}$-number, as wanted.

Let $M$ be the subgroup generated by all $\pi^{\prime}$-elements of $K \cap N$. Note that $M \neq 1$, otherwise $K \cap N$ would be a $\pi$-group and, since $|N: K \cap N|=|K N: K|$ divides $|G: K|$, which is a $\pi$-number too, then $N$ would be a $\pi$-group, a contradiction with Step 2. Let us prove that $M \unlhd G$. Let $\alpha$ be a generator of $M$, so $\left|\alpha^{G}\right|$ is $\pi^{\prime}$-number. Since $\left(|G: K|,\left|\alpha^{G}\right|\right)=1$ we have $G=K \mathbf{C}_{G}(\alpha)$ and hence, $\alpha^{G}=\alpha^{K} \subseteq K \cap N$. Therefore $\alpha^{G} \subseteq M$, as wanted.

Let $D=\left\langle x^{G}, y^{G}\right\rangle$. Notice that $D \unlhd G$ and $D \subseteq N$. Let $\alpha$ be a generator of $M$. As we have proved that $\left|\alpha^{G}\right|$ is $\pi^{\prime}$-number, then $\left(\left|\alpha^{G}\right|,\left|x^{G}\right|\right)=1$, so $G=\mathbf{C}_{G}(x) \mathbf{C}_{G}(\alpha)$. Thus, $x^{G}=x^{\mathbf{C}_{G}(\alpha)} \subseteq \mathbf{C}_{G}(\alpha)$ because $\alpha \in K$. The same happens for $y$, that is, $y^{G} \subseteq \mathbf{C}_{G}(\alpha)$, so we conclude that $[M, D]=1$.

We define $L=M D$ and we distinguish two cases. Assume first that $L<N$. Note that $x, y \in L \unlhd G$ and $L$ trivially satisfies the hypotheses of the theorem. By applying induction to $L$ we have in particular $L=\mathbf{O}_{\pi}(L) \times \mathbf{O}_{\pi^{\prime}}(L)$. Observe that the fact that $M \neq 1$ implies that $\mathbf{O}_{\pi^{\prime}}(L)>1$. Now, by definition of $M$, we have that $|K \cap N: M|$ is a $\pi$-number. As $|N: K \cap N|$ is also a $\pi$-number, it follows that $\left|N: \mathbf{O}_{\pi^{\prime}}(L)\right|$ is a $\pi$-number too. Then, $\mathbf{O}_{\pi^{\prime}}(L)=\mathbf{O}_{\pi^{\prime}}(N)$ is a Hall $\pi^{\prime}$-subgroup of $N$. We can apply Lemma 1 so as to conclude that $N=\mathbf{O}_{\pi}(N) \times \mathbf{O}_{\pi^{\prime}}(N)$ with $x, y \in \mathbf{O}_{\pi}(N)$. Since $\mathbf{O}_{\pi^{\prime}}(N)>1$, we apply the inductive hypotheses to $\mathbf{O}_{\pi}(N)<N$ and we deduce that $\mathbf{O}_{\pi}(N)$ is a quasiFrobenius group with abelian kernel and complement or $\mathbf{O}_{\pi}(N)=P \times A$ with $A \leq \mathbf{Z}(N)$ and $P$ is a $p$-group so the theorem is finished.

From now on, we assume that $L=N$ and let us see that $\mathbf{Z}(N)=1$. Otherwise, we take $\bar{N}=N / \mathbf{Z}(N)$ and $\bar{G}=G / \mathbf{Z}(N)$. If $\left|\bar{x}^{\bar{G}}\right|=1$, then $[\bar{x}, \bar{y}]=1$, and thus $[x, y] \in \mathbf{Z}(N)$. Since $(o(x), o(y))=1$, it is easy to prove that $[x, y]=1$, a contradiction. Analogously, we have $\left|\bar{y}^{\bar{G}}\right| \neq 1$. Consequently, $\bar{N}$ satisfies the assumptions of the theorem. By induction, we have $\bar{N}=\mathbf{O}_{\pi^{\prime}}(\bar{N}) \times \mathbf{O}_{\pi}(\bar{N})$ with $\bar{x}, \bar{y} \in \mathbf{O}_{\pi}(\bar{N})$ and $\mathbf{O}_{\pi}(\bar{N})$ is either a quasi-Frobenius group with abelian kernel 
and complement or $\bar{N}=\bar{P} \times \bar{A}$ with $\bar{A} \leqslant \mathbf{Z}(\bar{N})$ and $\bar{P}$ a $p$-group. In the latter case, $[\bar{y}, \bar{x}]=1$ which leads to a contradiction as we have seen before. So we are in the former case. It follows that $N=\mathbf{O}_{\pi^{\prime}}(N) \times \mathbf{O}_{\pi}(N)$ with $x, y \in \mathbf{O}_{\pi}(N)$ and by applying induction to $\mathbf{O}_{\pi}(N)<N$, we have the result. Therefore, $\mathbf{Z}(N)=1$. On the other hand, we have proved that $[M, D]=1$. Thus $M \cap D \subseteq \mathbf{Z}(N)=1$ and $N=M \times D$ with $x, y \in D$. Since $M \neq 1$, we can apply induction to $D$ and we get $D=\mathbf{O}_{\pi^{\prime}}(D) \times \mathbf{O}_{\pi}(D)$ with $x, y \in \mathbf{O}_{\pi}(D)$ and $\mathbf{O}_{\pi}(D)$ is a Frobenius group with abelian kernel and complement (notice that $\mathbf{Z}\left(\mathbf{O}_{\pi}(D)\right.$ ) $=1$ because $\mathbf{Z}(N)=1$ ). The $p$-group case cannot occur because $x$ and $y$ do not commute. Notice that if $M$ is $\pi^{\prime}$-group then the theorem is proved. Assume then that $M$ is not a $\pi^{\prime}$-group and we will obtain a contradiction. Let $s \in \pi$ such that $s$ divides $|M|$. We can assume that $s \in \pi_{x}$ (we proceed analogously if $s \in \pi_{y}$ ). Suppose that there exists an $s^{\prime}$-element $z \in M$ such that $\left|z^{M}\right|$ is divisible by $s$. Since $N$ is the direct product of $M$ and $D$, we have that $(z y)^{N}=z^{N} y^{N}$ is a non-trivial class of $N$ whose size is divisible by $s$ and by some prime of $\left|y^{N}\right| \neq 1$. This is not possible because $\left|(z y)^{G}\right|$ would have primes in $\pi_{x}$ and $\pi_{y}$. Thus, the class size of every $s^{\prime}$-element of $M$ is a $s^{\prime}$-number. It is known that $M=M_{1} \times S$ with $S \in \operatorname{Syl}_{s}(M)$. In this case, $\mathbf{Z}(S) \subseteq \mathbf{Z}(N)=1$, a contradiction.

Step 6. Conclusion in case $p=q$.

Let $K=\mathbf{C}_{G}(x) \cap \mathbf{C}_{G}(y)$ as in Step 2. Let $z$ be a $p^{\prime}$-element of $K \cap N$ and let us prove that $\left|z^{G}\right|$ is a $\pi^{\prime}$-number. Suppose that $s \in \pi$ is a prime divisor of $\left|z^{G}\right|$. We can assume for instance that $s \in \pi_{y}$, otherwise we proceed analogously. Since $\left|z^{G}\right|$ divides $\left|(z x)^{G}\right|$ we obtain that $s$ divides $\left|(z x)^{G}\right|$. On the other hand, we know by the proof of Step 2 that $q \in \pi_{x}$. Therefore, $\left|(z x)^{G}\right|$ is divisible by primes in $\pi_{x}$ and $\pi_{y}$, a contradiction. Consequently, $s \notin \pi$ and $\left|z^{G}\right|$ is a $\pi^{\prime}$-number, as wanted.

Let $T$ be the subgroup generated by all $p^{\prime}$-elements of $K \cap N$. We have that $T \neq 1$ because otherwise $K \cap N$ would be a $\pi$-group and this implies that $N$ is a $\pi$-group as in Step 5 , a contradiction. Let us prove that $T \unlhd G$. If $\alpha$ is a generator of $T$, we know that $\left|\alpha^{G}\right|$ is $\pi^{\prime}$-number. Then $\left(|G: K|,\left|\alpha^{G}\right|\right)=1$, so we have $G=K \mathbf{C}_{G}(\alpha)$ and $\alpha^{G}=\alpha^{K} \subseteq K \cap N$. Therefore, $\alpha^{G} \subseteq T$ as wanted.

Since the class size of every $p^{\prime}$-element of $T$ is a $p^{\prime}$-number then, by Lemma 2, $T=\mathbf{O}_{p}(T) \times \mathbf{O}_{p^{\prime}}(T)$. However, by definition of $T$, we have $\mathbf{O}_{p}(T)=1$, or equivalently $M=\mathbf{O}_{p^{\prime}}(T)$. Now, notice that if $s \in \pi$ and $s \neq p$, then the class size of every element of $T$ is an $s^{\prime}$-number so, it is well know that $T$ has a Sylow $s$-subgroup central and we can write $T=\mathbf{O}_{\pi}(T) \times \mathbf{O}_{\pi^{\prime}}(T)$. On the other hand, $|N: T|=|N: K \cap N||K \cap N: T|$ where $|N: K \cap N|=|K N: K|$ is a $\pi$-number and $|K \cap N: T|$ is a power of $p \in \pi$. Therefore $\mathbf{O}_{\pi^{\prime}}(T)=\mathbf{O}_{\pi^{\prime}}(N)$ and $\mathbf{O}_{\pi^{\prime}}(N)$ is a Hall $\pi^{\prime}$-subgroup of $N$. We have proved that the class size of every $p^{\prime}$-element of $N$ is a $\pi^{\prime}$-number, so by Lemma 1 , we have $N=\mathbf{O}_{\pi^{\prime}}(N) \times \mathbf{O}_{\pi}(N)$. We apply induction to $\mathbf{O}_{\pi}(N)<N$ and the proof is finished. 
We give an example showing that the converse of Theorem A is not true.

Example 1. We take the Special Linear group $H=\mathrm{SL}(2,5)$ which is a group of order 120 that acts Frobeniusly on $K=\mathbb{Z}_{11} \times \mathbb{Z}_{11}$. Let $P \in \operatorname{Syl}_{5}(H)$ and we consider $\mathbf{N}_{H}(P)$. Then, we define $N=K P$, which is trivially a normal subgroup of $G=K \mathbf{N}_{H}(P)$. We have that the set of the $G$-conjugacy class sizes of $N$ is $\{1,20,242\}$. Consequently, there are not two non-central $G$-classes of $N$ such that any non-central $G$-class of $N$ has size coprime with one of both.

Let us look at several examples illustrating Theorem A.

Example 2. We take the following groups from the library SmallGroups of GAP $G_{1}=\operatorname{Id}(324,8)$ and $G_{2}=\operatorname{Id}(168,44)$ that have the normal subgroups exposed now. The abelian 3-subgroup $P=\mathbb{Z}_{3} \times \mathbb{Z}_{3}$ and $A=\mathbb{Z}_{2} \times \mathbb{Z}_{2} \times \mathbb{Z}_{2}$, respectively. It follows that the set of conjugacy class sizes of $P$ is $\{1,2,3,3\}$ and the set of conjugacy class sizes of $A$ is $\{1,7\}$. We construct $N=P \times A$ and $G=G_{1} \times G_{2}$. We have that $N$ is a normal subgroup of $G$ and the set of $G$-conjugacy class sizes of $N$ is $\{1,2,3,7,14,21\}$ so $d\left(\Gamma_{G}(N)\right)=3$ and $N$ satisfies that it is the direct product of a 3-group and $A \leq \mathbf{Z}(N)$. Note that in this example it follows that $\mathbf{O}_{\pi^{\prime}}(N)=1$ and $\pi=\{2,3\}$.

Example 3. In order to illustrate the quasi-Frobenius case it is enough to consider any group $G$ and a normal subgroup $N=G$ such that $\Gamma(N)$ has two connected components. Thus, by applying Theorem of [3] we know that $N$ is a quasi-Frobenius group with abelian kernel and complement.

\section{Acknowledgements}

The results in this paper are part of the third author's Ph.D. thesis at the University Jaume I of Castellon. The research of the first and second authors is supported by the Valencian Government, Proyecto PROMETEOII/2015/011. The first author is also partially supported by Universitat Jaume I, grant P11B2012-05.

\section{References}

[1] A. Beltrán and M.J. Felipe, Prime powers as conjugacy class lengths of $\pi$-elements. Bull. Austral. Math. Soc. (69) (2004), 317-325.

[2] A. Beltrán, M.J. Felipe and C. Melchor, Graphs associated to conjugacy classes of normal subgroups in finite groups. J.Algebra, (443) (2015), 335348 .

[3] E.A. Bertram, M. Herzog and A. Mann, On a graph related to conjugacy classes of groups. Bull. London Math. Soc., 22 (6) (1990), 569-575. 
[4] A.R. Camina, Arithmetical conditions on the conjugacy class numbers of a finite group. J.London Math. Soc. (2) 5 (1972), 127-132.

[5] S. Dolfi, Arithmetical conditions of the length of the conjugacy classes in finite groups. 174, (3) (1995), 753-771.

[6] C. Casolo, S. Dolfi, The diameter of a conjugacy class graph of finite groups. Bull. London Math. Soc. 28 (1996), 141-148.

[7] L.S. Kazarin, On groups with isolated conjugacy classes, Izv. Vyssh. Uchebn. Zaved. Mat. (7) (1981), 40-45. 